\title{
Direct-write electron beam lithography in silicon dioxide at low energy
}

Arnaud Beaumont, Christian Dubuc, Jacques Beauvais, and Dominique Drouin

Citation: Journal of Vacuum Science \& Technology B, Nanotechnology and Microelectronics: Materials, Processing, Measurement, and Phenomena 28, 940 (2010); doi: 10.1116/1.3478304

View online: http://dx.doi.org/10.1116/1.3478304

View Table of Contents: http://avs.scitation.org/toc/jvb/28/5

Published by the American Vacuum Society

Contact Hiden Analytical for further details: W www.HidenAnalytical.com E info@hiden.co.uk

CLICK TO VIEW our product catalogue

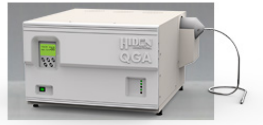

Gas Analysis

dynamic measurement of reaction gas stream catalysis and thermal analysis

molecular beam studies

dissolved species probes

fermentation, environmental and ecological studies

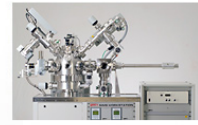

Surface Science UHVTPD , SIMS

, end point detection in ion beam etch elemental imaging - surface mappin

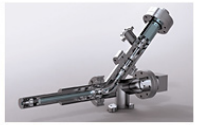

Plasma Diagnostics plasma source characterization etch and deposition process reaction , kinetic studies analysis of neutral and radical species

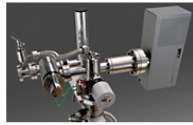

Vacuum Analysis - partial pressure measurement and contro of process gases , reactive sputter process control , vacuum diagnostics - vacuum coating process monitoring 


\title{
Direct-write electron beam lithography in silicon dioxide at low energy
}

\author{
Arnaud Beaumont ${ }^{\mathrm{a})}$ \\ Department of Electrical Engineering, Universite de Sherbrooke, Sherbrooke, QC J1K2R1, Canada \\ Christian Dubuc \\ OSEMI Canada, Sherbrooke, QC J1J1G9, Canada \\ Jacques Beauvais and Dominique Drouin \\ Department of Electrical Engineering, Universite de Sherbrooke, Sherbrooke, QC J1K2R1, Canada
}

(Received 26 January 2010; accepted 20 July 2010; published 31 August 2010)

\begin{abstract}
Electron beam lithography in silicon dioxide has been investigated with energies ranging from 0.5 up to $6 \mathrm{keV}$. The etch ratio of $\mathrm{SiO}_{2}$ has been studied and interpreted with regard to the limited penetration of electrons at such low energies. Monte Carlo simulations have been carried out to investigate the depth of penetration and the density of energy absorbed by $\mathrm{SiO}_{2}$. The etch ratio is also shown to depend on the dilution of the developer (a buffered hydrofluoric acid diluted in water). Finally, a possible application of low energy direct writing in silicon dioxide is described for the control of damascene processes, enabling the fabrication of nanodevices embedded in an insulator. (ㅇ 2010 American Vacuum Society. [DOI: 10.1116/1.3478304]
\end{abstract}

\section{INTRODUCTION}

Electron beam lithography (EBL) is widely used for the fabrication of nanostructures. Because they feature high sensitivity and high contrast, organic resists have become the standards for transferring nanoscale patterns into different functional layers. It is possible to use inorganic materials as an alternative to organic resist patterning. Their advantage is that they feature high resolution while enabling direct writing into materials with useful properties (e.g., electrical or optical). However, inorganic resists are mostly less sensitive than organic ones, and the throughput loss related to this issue restricts the applications of these materials to research purposes. The use of low energy EBL somewhat increases the speed of the patterning of inorganic materials but not enough to compensate the intrinsically high doses required to alter their properties. However, low energy electron beams may be attractive for direct writing of nanoscale research prototypes because the penetration of electrons is limited to a few nanometers under the surface of the target material. Moreover, it is able to reach nanoscale resolution, thanks to recent improvements in EBL systems enabled by the spread of field emission guns providing electron beams of less than $5 \mathrm{~nm}$ in diameter with acceleration voltages of a few $\mathrm{keV}$. Early studies on inorganic resists dealt with the use of $\mathrm{SiO}_{2}$ because it is the original insulator of Si-based microelectronics. Direct writing in silicon dioxide was first demonstrated by O'Keeffe and Handy in $1968,{ }^{1}$ but it has attracted much attention later in the 1990s after Allee and Broers showed that this technique had nanometer scale capabilities. ${ }^{2}$ A significant amount of data has been published at that time for high energy $(>50 \mathrm{keV})$ direct patterning of $\mathrm{SiO}_{2} \cdot{ }^{2-8}$ The best result reported in terms of resolution is an array of $5 \mathrm{~nm}$ wide lines with a $10.8 \mathrm{~nm}$ pitch achieved with an energy of $300 \mathrm{keV}$ and optimized lithography conditions. ${ }^{5}$ However,

${ }^{a)}$ Electronic mail: arnaud.beaumont@usherbrooke.ca very little data are available at low energy, ${ }^{1,9}$ whereas it considerably fastens the process time and reduces eventual irradiation damage in the substrate due to the penetration of energetic electrons. This paper consequently reports on etch ratio (ER) and sensitivity of $\mathrm{SiO}_{2}$ as a direct writing material at low acceleration voltage $(<6 \mathrm{kV})$. An emphasis is also put on how to use it to monitor the progress of chemical mechanical planarization (CMP) steps in damascene processes.

\section{MATERIALS AND METHODS}

Undoped $\mathrm{Si}$ wafers were oxidized for $25 \mathrm{~min}$ at $900{ }^{\circ} \mathrm{C}$ in pure oxygen, providing an $80 \mathrm{~nm}$ thick $\mathrm{SiO}_{2}$ layer. EBL was performed on $1 \times 1 \mathrm{~cm}^{2}$ samples in a LEO Supra $55 \mathrm{VP}$ scanning electron microscope (SEM) equipped with a pattern generator software (NPGS 9.0 by J. Nabity). Acceleration voltage was varied from 1 to $6 \mathrm{kV}$. Patterns consisted in 40 $\times 10 \mu \mathrm{m}^{2}$ rectangles exposed with area doses ranging from 0.02 to $0.10 \mathrm{C} \mathrm{cm}^{-2}$. High dose electron beam exposure implies the deposit of thin organic compounds at the surface of the samples due mainly to contamination of the SEM chamber. Therefore, a pure $\mathrm{O}_{2}$ plasma was applied to the samples in a barrel-asher (Tegal Plasmaline 211) with a power of 50 $\mathrm{W}$ for $2 \mathrm{~min}$ before development. The developer is a buffered oxide etchant $\left(6: 1 \mathrm{NH}_{4} \mathrm{~F}: \mathrm{HF}\right)$ solution diluted in water. The HF concentration was typically $2 \%$ in volume, but this ratio was also varied from $1 \%$ to $5 \%$ in volume in order to investigate its influence on results. The depth of $40 \times 10 \mu \mathrm{m}^{2}$ trenches was measured with regard to the development time by successive profilometer measurements (Tencor P-1). The thickness of nonexposed areas was measured with a Woollam Alpha-SE ellipsometer before and after each etch step to deduce remaining oxide thickness. 


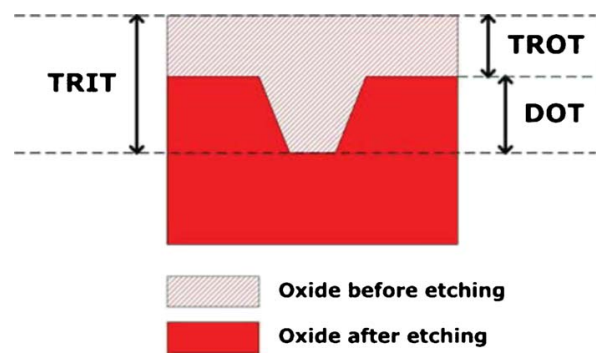

FIG. 1. (Color online) Schematic of the variables involved in the calculation of oxide etch ratios after development in HF-based solutions.

\section{RESULTS AND DISCUSSION}

Irradiating a stoichiometric $\mathrm{SiO}_{2}$ layer with energetic electrons is suggested to modify the density and the stoichiometry of the oxide layer. ${ }^{1,2}$ Thus, it produces a $\mathrm{SiO}_{x}(x$ $<2$ ), which shows a higher etch rate in HF-based aqueous solutions. ${ }^{1,4}$ The ratio between the etch rate of exposed areas and nonexposed areas is hereafter designed as the ER. In this paper, ER was calculated with the relation ER $=$ TRIT/TROT, where TRIT is the thickness removed in the trenches and TROT is the thickness removed outside the trenches according to the conventions sketched in Fig. 1. Since TRIT $=$ DOT + TROT, where DOT is the depth of trenches, it is more convenient to use the relationship ER $=1+$ DOT $/$ TROT because it involves two quantities actually measurable after lithography and development: DOT is measured by profilometry and TROT is measured by ellipsometry.

\section{A. Etch ratio measurements and analysis}

In Fig. 2, the $\mathrm{SiO}_{x} / \mathrm{SiO}_{2}$ ER is shown as a function of the area dose for different electron beam energies $\left(E_{b}\right)$. When the dose is increased, ER first increases and then saturates. The saturation dose is proportional to $E_{b}$. For $E_{b}$ greater than 3 $\mathrm{keV}$, the saturation dose is not reached due to the dose range considered in this study. Maximal ER values are about 3 for

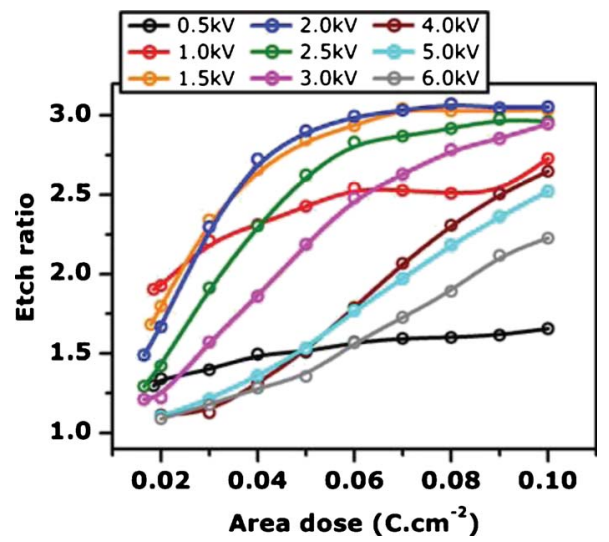

FIG. 2. (Color online) Etch ratio measured by profilometry for several EBL energies ranging from 0.5 to $6 \mathrm{kV}$ as a function of the area dose. Measurements were performed on $40 \times 10 \mu \mathrm{m}^{2}$ structures developed in a $\mathrm{HF}: \mathrm{NH}_{4} \mathrm{~F}: \mathrm{H}_{2} \mathrm{O}$ (1:6:93) solution (HF concentration of $1 \%$ in volume) for $180 \mathrm{~s}$.

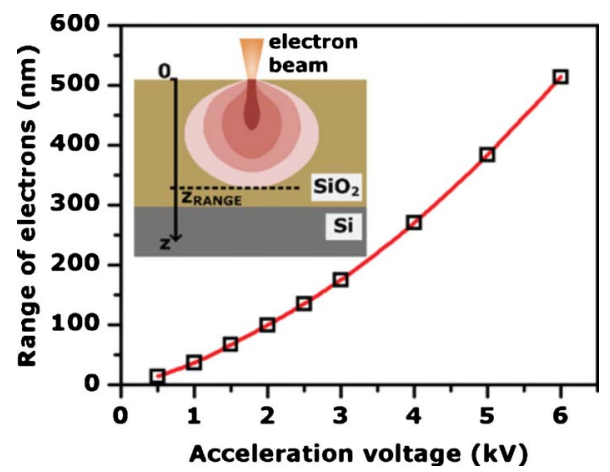

FIG. 3. (Color online) Electrons range simulated with the software CASINO 2.42 as a function of the acceleration voltage in an $80 \mathrm{~nm}$ thick $\mathrm{SiO}_{2}$ layer over a Si substrate. The inset illustrates how the stopping range is defined: the areas represented under the electron beam with shades ranging from light to dark represent the increasing ratio of energy transferred from incident nonbackscattered electrons to the target material.

energies of $1.5,2$, and $2.5 \mathrm{keV}$. This is in agreement with values previously reported for higher energies, ${ }^{1,3,4,6}$ indicating that there is a continuity in the physical behavior of $\mathrm{SiO}_{2}$ irradiated with electrons from $300 \mathrm{keV}$ down to $1.5 \mathrm{keV}$. As a consequence, $1.5 \mathrm{kV}$ is an acceleration voltage of choice for direct-write EBL since it reaches the maximal ER for the lowest area dose $\left(0.07 \mathrm{C} \mathrm{cm}^{-2}\right)$. In other words, an energy of $1.5 \mathrm{keV}$ enables the best sensitivity, commonly defined to be the ER/dose ratio. However, ER is found to saturate at 1.6 and 2.5 for energies of 0.5 and $1 \mathrm{keV}$, respectively. These values may be interpreted by considering that the etch time was fixed at $180 \mathrm{~s}$. Under these conditions, ER values are related to the electron range. Indeed, during EBL, the electrons lose their energy in a limited volume of material (named as the interaction volume and hereafter abbreviated as EMIV for electron-material interaction volume). If the etch time is longer than the time needed to completely etch the EMIV, a removal regime with a ratio of 1 takes place. This consequently decreases the final ER, which is then an average over the etch period of the ER inside the EMIV and $\mathrm{ER}=1$ outside the EMIV. This interpretation is made plausible by the observation that the decrease in ER occurs with the lowest energies. Indeed, with the highest energies, the EMIV is expected to be too deep to be completely etched with the diluted HF solution and the etch time used in this study. A further validation was carried out by simulating the electron range using the Monte Carlo software CASINO 2.42 as a function of the acceleration voltage used for the lithography. ${ }^{10}$ The simulations are shown in Fig. 3, where the depth of the EMIV, i.e., the stopping range (" $z$ range") of the electrons, is defined as the depth at which $99.9 \%$ of the energy of nonbackscattered electrons is absorbed by the material. As expected, the stopping range increases when the acceleration voltage increases. For doses high enough to reach saturation plateaus in Fig. 2, ER is assumed to be constant and equal to $\mathrm{ER}_{\max }$, the maximal ratio measured for energies of $1.5,2$, and $2.5 \mathrm{kV}$. The mean ER may then be evaluated by the relation 


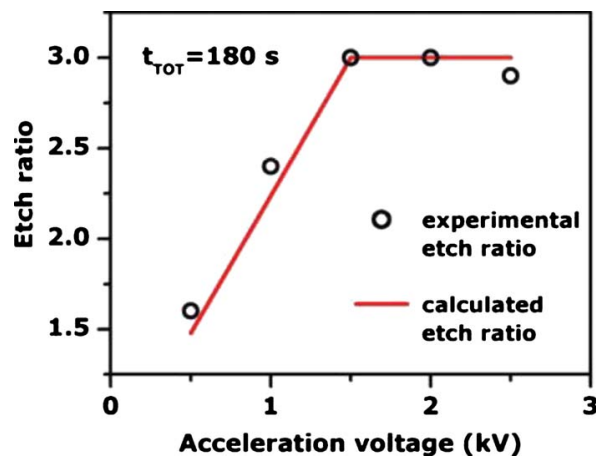

FIG. 4. (Color online) Mean etch ratio extracted from the plateaus of Fig. 2 plotted versus the acceleration voltage and compared to values calculated by Eq. (1).

$$
\overline{\mathrm{ER}}=\frac{\int_{0}^{t_{\mathrm{tot}}} R_{I} \cdot d t}{\int_{0}^{t_{\mathrm{tot}}} R_{O} \cdot d t}=\frac{\int_{0}^{t_{\mathrm{range}}} \mathrm{ER}_{\mathrm{max}} \cdot R_{O} d t+\int_{t_{\mathrm{range}}}^{t_{\mathrm{tot}}} R_{O} d t}{R_{O} \cdot t_{\mathrm{tot}}},
$$

where $R_{I}$ is the etch rate inside the EMIV, $R_{O}$ is the etch rate outside the EMIV, $t_{\text {range }}$ is the time needed to etch the EMIV, and $t_{\text {tot }}$ is the total etching time. Mean ERs calculated with this relation are plotted in Fig. 4 versus the acceleration voltage, and a good agreement is found with experimental data. This confirms that for acceleration voltages of 0.5 and $1 \mathrm{kV}$, the EMIV was completely etched, and some underlying nonirradiated $\mathrm{SiO}_{2}$ was also etched. This is why the acceleration voltage has to be carefully chosen with regard to the depth targeted by the lithography process. This choice may be done with Monte Carlo simulations of the range of electrons for a given acceleration voltage. For instance, the energy absorbed during a lithography performed with an acceleration voltage of $1.5 \mathrm{kV}$ was simulated with CASINO as a function of the depth in an $80 \mathrm{~nm}$ thick $\mathrm{SiO}_{2}$ layer (Fig. 5(a)). The range of the electrons was extracted, and then the etch rate was monitored by ellipsometry during the development of patterns processed with this acceleration voltage (Fig. 5(b)). As expected, ER measured by profilometry was found superior to 1 until the etched depth equals the electron range $(65 \mathrm{~nm}$ in

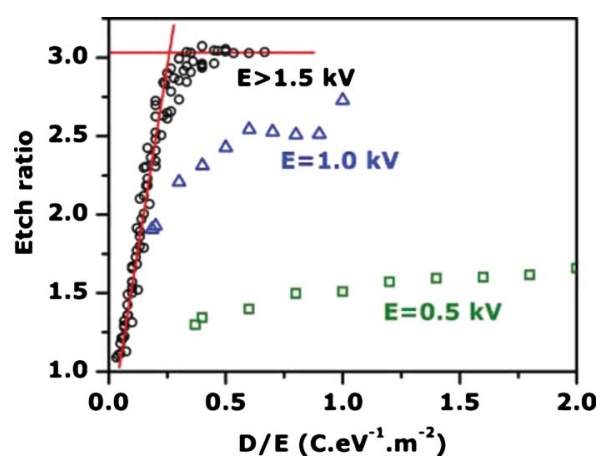

FIG. 6. (Color online) Etch ratio plotted versus the dose/energy ratio. Shapes of data points correspond to the acceleration voltage used for lithography: $0.5 \mathrm{kV}$ (squares), $1 \mathrm{kV}$ (triangles), and superior to $1.5-6 \mathrm{kV}$ (circles).

this case). Further development of the patterns resulted in a ratio equal to 1 , which means that no additional depth could be obtained: In this case, the maximal depth of the patterns is $35 \mathrm{~nm}$.

\section{B. Relation between dose, energy, and etch ratio}

From the data presented in Fig. 2, a consistent relation between dose $(D)$, energy $(E)$, and resulting ER was found. Indeed, as shown in Fig. 6, for energies above $1.5 \mathrm{keV}$, an ER vs $D / E$ graph results in a straight line $(D / E$ $<0.2 \mathrm{C} \mathrm{eV}^{-1} \mathrm{~m}^{-2}$ ) followed by a plateau. For lower energies, the trend is similar, but since the EMIV is completely removed the etch rate is lowered, as discussed in Sec. I. The plateau observed for $E>1.5 \mathrm{kV}$ is related to the maximal ER (around 3) than can be reached by the lithography in $\mathrm{SiO}_{2}$. On the other hand, the straight line suggests that an increase in energy requires a linear increase in dose to obtain a given ER. This trend has already been reported experimentally in various resists such as ZEP-520, ${ }^{11}$ hydrogen silsesquioxane, ${ }^{12}$ calixarene, ${ }^{13}$ and polystyrene. ${ }^{14}$ This linearity is generally attributed to the relation existing between the dose, the energy, and the ER through the concept of stopping power. In Ref. 15, Bethe defined the stopping power, i.e., the energy loss by unit length in the depth direction $z$, as
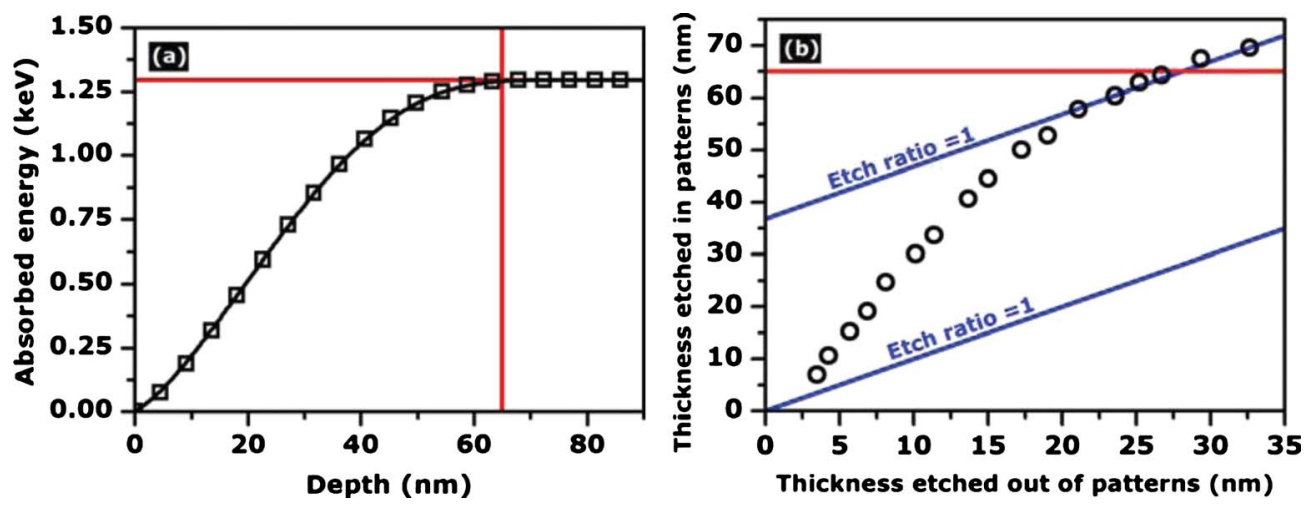

FIG. 5. (Color online) (a) Plot of the total absorbed energy simulated with an acceleration voltage of $1.5 \mathrm{kV}$ versus depth. (b) Thickness removed in the patterns plotted versus the thickness etched out of the patterns. 

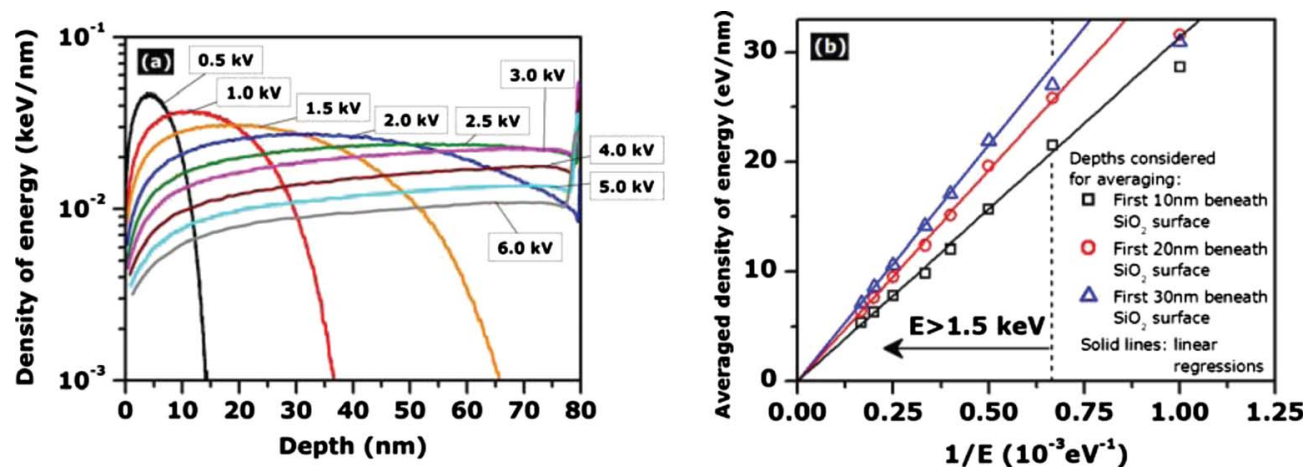

FIG. 7. (Color online) (a) Density of energy absorbed in $\mathrm{SiO}_{2}$ as a function of the depth and (b) plot of the averaged density of energy versus the inverse of the incident energy.

$$
\frac{d E}{d z}=7.85 \times 10^{4} \frac{Z \rho}{A E} \ln \left(\frac{1.166 E}{J}\right)(\text { in } \mathrm{eV} / \mathrm{nm}),
$$

where $E$ is the energy of the electron beam (in eV), $z$ is the depth in the target material, $Z$ is the atomic number, $\rho$ is the density of the material (in $\mathrm{g} / \mathrm{cm}^{3}$ ), $A$ is the atomic mass, and $J$ is the excitation energy (in eV). This relation is the nonrelativistic form of the Bethe stopping power, known to be relevant for energies inferior to $30 \mathrm{keV}$ as it is the case in this study. However, its validity is questionable for energies below $10 \mathrm{keV},{ }^{16}$ and it is worth noting that the logarithm becomes negative if $E$ is smaller than $J / 1.166$. This led Joy and Luo to modify Bethe's relation empirically in order to address low energy electrons. ${ }^{17}$ But the trajectories of low energy electrons are also strongly affected by forward scattering. Then, this has to be taken into account to predict the density of energy transferred to the resist, which may be achieved with Monte Carlo simulators. Figure 7(a) shows simulations of the energy density transferred to $\mathrm{SiO}_{2}$ calculated using CASINO (with Joy and Luo's stopping power) for energies ranging from 0.5 to $6 \mathrm{kV}$. The energy density was averaged over the first few tens of nanometers beneath the $\mathrm{SiO}_{2}$ surface. These average values were found to be inversely proportional to the energy for $E>1.5 \mathrm{keV}$, as illustrated in Fig. 7(b) for the first 10, 20, and $30 \mathrm{~nm}$ beneath the $\mathrm{SiO}_{2}$ surface. Similar results may be found by averaging over larger depths, but since the range of low energy electrons would then be reached the results would only be valid for high energies. This also explains why in Fig. 7(b) the linearity is lost for the lowest energies (i.e., the highest $1 / E$ ratios) and more clearly when averaging over the first $30 \mathrm{~nm}$ beneath the $\mathrm{SiO}_{2}$ surface. The energy absorbed in the $\mathrm{SiO}_{2}$ is also proportional to the dose, i.e., the number of electrons incident per unit area of material. Thus, the density of energy loss per unit volume in the EMIV is linearly related to the dose/energy ratio:

$$
\frac{d^{3} E}{d x d y d z} \propto \frac{D}{E},
$$

$D$ being the dose and $d x d y d z$ a unit volume of material in the EMIV. Consequently, the shape of the curve presented in Fig. 6 is correctly interpreted if one assumes that ER is linear to the density of energy loss per unit volume and saturates when it reaches $E R_{\max }$. Such a graph is a helpful tool for electron beam lithography since a trade-off has to be found between the dose (i.e., the lithography processing time) and the energy, which has to be chosen according to the targeted depth of the patterns, on one hand, and to the targeted resolution, on the other hand. Indeed, in current SEMs, the lateral resolution of e-beam lithography is better when the acceleration voltage is high. However, a careful choice of the acceleration voltage for the fabrication of patterns leads to a wide range of available $D / E$ ratios, which means a reasonable range of ERs. The usefulness of the $D / E$ relationship lies in the fact that it is far more convenient to change the dose (with the exposure dwell time during the lithography) than the acceleration voltage (which requires new settings for the scanning electron microscope).

\section{Effect of HF dilution}

In the previous sections, the development steps were performed with a buffered HF solution diluted in water $(2 \%$ in volume). In order to check the effect of the dilution of the developer on ER, the dilution was varied from $1 \%$ up to $5 \%$ in volume while keeping other parameters fixed. The results are presented in Fig. 8, showing that ER decreases when the concentration is increased. The plateau on the left hand of

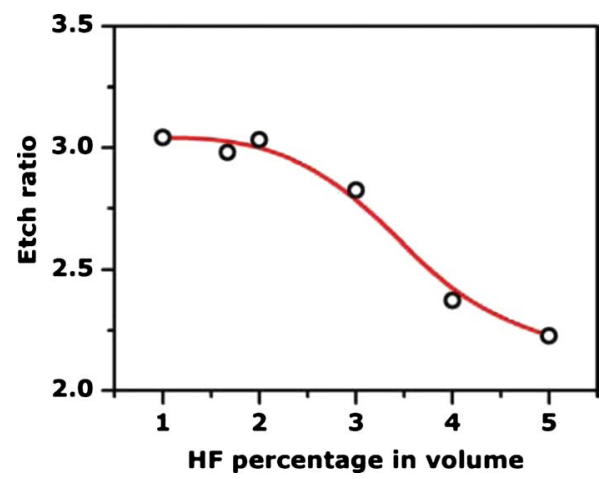

FIG. 8. (Color online) Etch ratio versus the HF percentage in volume of the developer. Electron beam lithography was performed with an acceleration voltage of $1.5 \mathrm{kV}$ and a dose of $0.1 \mathrm{C} \mathrm{cm}^{-2}$. 
the curve suggests that further dilution would not lead to a further increase in ER. This trend is attributed to the complex dissociation of HF in water, which is known to result in the coexistence of many different species of ions with various concentrations as a function of the developer dilution. This would indicate that the maximum ER observed in this study (and similar to most of the values reported in the literature) is related to the predominance of particular ions in the solution, appearing only in largely diluted BHF solutions. ${ }^{6}$

\section{Application to stair-shaped end point structures for damascene processes}

Processes based on $\mathrm{SiO}_{2}$ direct-write electron beam lithography have already been proposed for the fabrication of nanodevices. Most of them are based on the fabrication of thin $\mathrm{SiO}_{2}$ hard masks useful to dry etch the underlying silicon $^{18}$ or selectively growing silicon nanowires by epitaxy. ${ }^{19}$ Although the use of low energy electrons decreases the dose required for the fabrication of useable structures, direct write remains several orders of magnitude slower than patterning equivalent areas by means of organic resists. Therefore, its use is limited to the fabrication of research nanodevices, which do not require fast processes but could benefit from the possibility to fabricate in one step patterns with various depths and nanometer resolution. More recently, it was also used in a nanodamascene process delivering single electron transistors (SETs) operating at room temperature. $^{20,21}$ In brief, the electrodes of the SETs are elaborated as follows: Thin trenches are first fabricated in a $\mathrm{SiO}_{2}$ layer, and some metal is then deposited onto the entire sample surface. A CMP step finally removes the metal outside the trenches, but these remain filled with metal, constituting metallic nanowires embedded in the oxide. As discussed above, direct writing enables one to control the depth of patterns in $\mathrm{SiO}_{2}$ by choosing an appropriate ER for each pattern. This is achieved by setting the dose/energy ratio according to an experimental ER vs $D / E$ graph (Fig. 6). Because this technique enables us to create patterns of chosen depth directly in a $\mathrm{SiO}_{2}$ layer, it was integrated into a damascene process to create metal-filled patterns of various depths. This study was driven by the need of end point detection in the nanodamascene process. Figure 9 illustrates the principle of the damascene process for the elaboration of stair-shaped structures. It consists in subsequently patterning a $\mathrm{SiO}_{2}$ layer, depositing a metal over the entire sample surface (Fig. 9(a)) and then performing a CMP step until reaching the oxide surface (Fig. 9(b)). This technique is of practical interest to fabricate visual indicators in the aim to monitor the progress of the CMP step in damascene processes. Indeed, the nanostructures elaborated by nanodamascene are assumed to have the same depth as the micrometric stair-shaped structures, enabling for monitoring the progress of the CMP step by a simple observation with an optical microscope. In Fig. 10(a), an optical micrograph of four single electron transistors is shown with a stair-shaped structure in the center. Figure 10(b) illustrates the cross-sectional view along the dotted line in Fig. 10. The end point structure
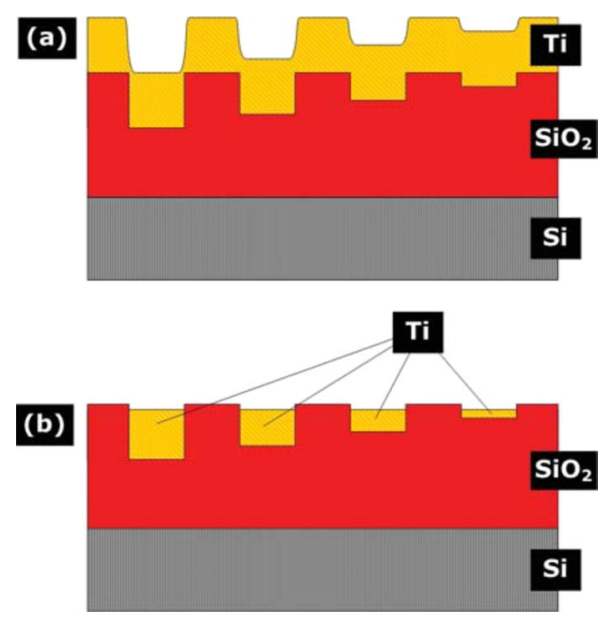

FIG. 9. (Color online) Schematic illustrating the process used for the fabrication of stair-shaped structures. After $\mathrm{SiO}_{2}$ patterning and metal deposition (a), metal-filled trenches are revealed by CMP (b).

consists of the numbers 1,2 , and 3 in the center of the optical micrograph. Here, the shallower mark (1) positioned at the left hand side of 2 was removed during the CMP step. Pattern 2 is not clearly visible since its depth was chosen as the end point of the CMP. Pattern 3 appears very brightly and has the same color as the electrodes of the devices, showing that its state is representative of the titanium thickness re-

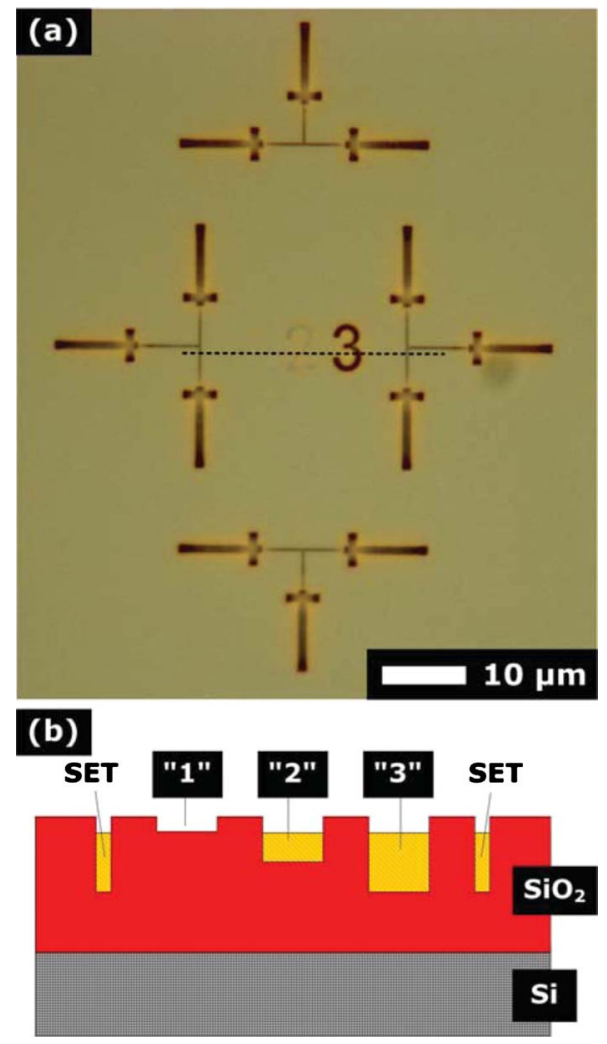

FIG. 10. (Color online) (a) Optical micrograph of four single electron transistors surrounding a three-stair damascene end point. (b) Cross-sectional sketch along the dotted line in the optical micrograph illustrating the depth of the patterns. 
maining in the trenches constituting the single electron transistors. Although such end point detection is not accurate enough to control the titanium thickness in the trenches of the devices, it constitutes a convenient optical test during CMP since it may be quickly performed after each polishing step. The progress of the process is further examined by resistivity measurements at the surface of the sample to confirm the absence of metal out of the patterns. Moreover, at least one of the four structures is simply a metal line used as a short-circuit test structure rather than a complete single electron transistor. Measurements of the resistivity of the leads are then performed to evaluate the progress of titanium removal in neighbor single electron devices.

\section{SUMMARY}

The etch ratio of $\mathrm{SiO}_{2}$ as an inorganic resist for electron beam direct writing has been studied at low energy for which the penetration of electrons is limited. ER has been shown to be linear with the dose/energy ratio, which is well explained by Monte Carlo simulations of the energy density absorbed in silicon dioxide during lithography. The dilution of the buffered HF developer is also an important parameter to predict ER after development. In our study, the lowest HF concentration $(<2 \%$ in volume) led to the highest ER (around 3). Direct-write electron beam lithography into $\mathrm{SiO}_{2}$ appears as a useful technique to create patterns with chosen depths although its low speed confines it to research purposes. The control is achieved by choosing an appropriate ER for each pattern through the setting of the dose/energy ratio according to an experimental ER vs $D / E$ graph. This opens the way to various applications in research; among them the creation of end point structures for critical CMP processes is described in this paper.

\section{ACKNOWLEDGMENTS}

The authors wish to thank the cleanroom staff of Universite de Shebrooke and especially Jean Beerens, Guillaume Bertrand, Mélanie Cloutier, Michael Lacerte, Pierre
Lafrance, Pierre Langlois, and Caroline Roy for technical support and fruitful discussions. They are also grateful to the National Sciences and Engineering Research Council of Canada (NSERC), the Fonds Québécois de la Recherche sur la Nature et les Technologies (FQRNT), and NanoQuébec for financial support.

${ }^{1}$ T. W. O'Keeffe and R. M. Handy, Solid-State Electron. 11, 261 (1968).

${ }^{2}$ D. R. Allee and A. N. Broers, Appl. Phys. Lett. 57, 2271 (1990).

${ }^{3}$ X. Pan, D. R. Allee, A. N. Broers, Y. S. Tang, and C. W. Wilkinson, Appl. Phys. Lett. 59, 3157 (1991).

${ }^{4}$ X. Pan and A. Broers, J. Appl. Phys. 71, 6189 (1992).

${ }^{5}$ X. Pan and A. N. Broers, Appl. Phys. Lett. 63, 1441 (1993).

${ }^{6}$ H. Hiroshima and M. Komuro, Jpn. J. Appl. Phys., Part 1 32, 6153 (1993).

${ }^{7}$ M. Komuro, H. Hiroshima, S. Haraichi, K. Ishii, T. Wada, and S. Okayama, Microelectron. Eng. 30, 411 (1996).

${ }^{8}$ T. Wada, S. Haraichi, K. Ishii, H. Hiroshima, M. Komuro, and S. M. Gorwadkar, J. Vac. Sci. Technol. A 16, 1430 (1998).

${ }^{9}$ C. Dubuc, J. Beauvais, and D. Drouin, IEEE Trans. Nanotechnol. 7, 68 (2008).

${ }^{10}$ D. Drouin, A. Couture, D. Joly, X. Tastet, V. Aimez, and R. Gauvin, Scanning 29, 92 (2007).

${ }^{11}$ H. Yang, L. Fan, A. Jin, Q. Luo, C. Gu, and Z. Cui, Proceedings of the First IEEE International Conference on Nano/Micro Engineered and Molecular Systems, 2006, p. 391.

${ }^{12}$ H. Yang, A. Jin, Q. Luo, C. Gu, Z. Cui, and Y. Chen, Microelectron. Eng. 83, 788 (2006).

${ }^{13}$ A. Tilke, M. Vogel, F. Simmel, A. Kriele, R. H. Blick, H. Lorenz, D. A. Wharam, and J. P. Kotthaus, J. Vac. Sci. Technol. B 17, 1594 (1999).

${ }^{14}$ S. Manako, J.-i. Fujita, Y. Ochiai, E. Nomura, and S. Matsui, Jpn. J. Appl. Phys., Part 1 36, 7773 (1997).

${ }^{15}$ H. Bethe, Ann. Phys. 397, 325 (1930).

${ }^{16}$ S. Tanuma, C. J. Powell, and D. R. Penn, Surf. Interface Anal. 37, 978 (2005).

${ }^{17}$ D. Joy and S. Luo, Scanning 11, 176 (1989).

${ }^{18}$ T. Tsutsumi, K. Ishii, H. Hiroshima, S. Hazra, M. Yamanaka, I. Sakata, H. Taguchi, E. Suzuki, and K. Tomizawa, J. Vac. Sci. Technol. B 18, 2640 (2000).

${ }^{19}$ N. Miyata, H. Watanabe, and M. Ichikawa, J. Vac. Sci. Technol. B 17, 978 (1999).

${ }^{20}$ C. Dubuc, J. Beauvais, and D. Drouin, Appl. Phys. Lett. 90, 113104 (2007).

${ }^{21}$ A. Beaumont, C. Dubuc, J. Beauvais, and D. Drouin, IEEE Electron Device Lett. 30, 766 (2009). 